\title{
Dirac particles' tunneling from five-dimensional rotating black strings influenced by the generalized uncertainty principle
}

\author{
Deyou Chen ${ }^{\text {a }}$ \\ Institute of Theoretical Physics, China West Normal University, Nanchong 637009, China
}

Received: 28 October 2013 / Accepted: 5 December 2013 / Published online: 23 January 2014

(C) The Author(s) 2014. This article is published with open access at Springerlink.com

\begin{abstract}
The standard Hawking formula predicts the complete evaporation of black holes. Taking into account the effects of quantum gravity, we investigate the tunneling of fermions from a five-dimensional rotating black string. The temperature is not only determined by the string, but also affected by the quantum numbers of the emitted fermion and the effect of the extra spatial dimension. The quantum correction slows down the increase of the temperature, which naturally leads to a remnant in the evaporation.
\end{abstract}

\section{Introduction}

The semi-classical tunneling method is an effective way to describe Hawking radiation [1,2]. Using this method, the tunneling behavior of massless particles across the horizon was adequately described in $[3,4]$. In this research, a varying background spacetime was taken into account. The tunneling rate was related to the change of the BekensteinHawking entropy and the temperature was higher than the standard Hawking temperature. In the former research, the standard temperatures were derived [5-10], which implies complete evaporation of the black holes. Thus the varying background spacetime accelerates the black holes' evaporation. This result was also demonstrated in other complicated spacetimes [11-15]. Extending this work to massive particles, the tunneling radiation of general spacetimes was investigated in [16-18]. The same result was derived by the relation between the phase velocity and the group velocity.

In $[19,20]$, the standard Hawking temperatures were recovered by fermions tunneling across the horizons. In the derivation, the action of the emitted particle was derived by the Hamilton-Jacobi equation [21]. This derivation is based on the method of complex path analysis [22]. In this method, we do not need the consideration that the particle moves along

a e-mail: ruijianchen@gmail.com; dyouchen@gmail.com the radial direction [23-26]. This is different from the work of Parikh and Wilczek [3,4].

The tunneling radiation beyond the semi-classical approximation was discussed in [27-29]. Their ansatz is also based on the Hamilton-Jacobi method. The key point is to expand the action in powers of $\hbar$. Using the expansion, one can get the quantum corrections over the semi-classical value. The corrected temperature is lower than the standard Hawking temperature. The higher order correction entropies were derived by the first law of black-hole thermodynamics.

Taking into account the effects of quantum gravity, the semi-classical tunneling method was reviewed in the recent work [30-32]. In [30,31], the tunneling of massless particles through the quantum horizon of a Schwarzschild black hole was investigated by the influence of the generalized uncertainty principle (GUP). Through the modified commutation relation for the radial coordinate, the conjugate momentum and the deformed Hamiltonian equation, the radiation spectrum was derived including the quantum correction. The thermodynamic quantities were discussed. In the fermionic fields, with the consideration of the effects of quantum gravity, the generalized Dirac equation in curved spacetime was derived by the modified fundamental commutation relation [33], which is [32]

$$
\begin{aligned}
& {\left[i \gamma^{0} \partial_{0}+i \gamma^{i} \partial_{i}\left(1-\beta m^{2}\right)+i \gamma^{i} \beta \hbar^{2}\left(\partial_{j} \partial^{j}\right) \partial_{i}\right.} \\
& \quad+\frac{m}{\hbar}\left(1+\beta \hbar^{2} \partial_{j} \partial^{j}-\beta m^{2}\right) \\
& \left.\quad+i \gamma^{\mu} \Omega_{\mu}\left(1+\beta \hbar^{2} \partial_{j} \partial^{j}-\beta m^{2}\right)\right] \psi=0 .
\end{aligned}
$$

This derivation is based on the existence of a minimum measurable length. This length can be realized in a model of the GUP

$\Delta x \Delta p \geq \frac{\hbar}{2}\left[1+\beta(\Delta p)^{2}+\beta\langle p\rangle^{2}\right]$,

where $\beta=\beta_{0} \frac{l_{p}^{2}}{\hbar^{2}}$ is a small value, $\beta_{0}<10^{34}$ is a dimensionless parameter and $l_{p}$ is the Planck length. Equation (2) 
was derived by the modified Heisenberg algebra $\left[x_{i}, p_{j}\right]=$ $i \hbar \delta_{i j}\left[1+\beta p^{2}\right]$, where $x_{i}$ and $p_{i}$ are position and momentum operators, defined, respectively, as $[33,34]$

$x_{i}=x_{0 i}$,

$p_{i}=p_{0 i}\left(1+\beta p_{0}^{2}\right)$,

$p_{0}^{2}=\sum p_{0 j} p_{0 j}, x_{0 i}$ and $p_{0 j}$ satisfy the canonical commutation relations $\left[x_{0 i}, p_{0 j}\right]=i \hbar \delta_{i j}$. Thus the minimal position uncertainty is gotten as

$\Delta x=\hbar \sqrt{\beta} \sqrt{1+\beta\langle p\rangle^{2}}$,

which means that the minimum measurable length is $\Delta x_{0}=$ $\hbar \sqrt{\beta}$ [33]. For $\Delta x_{0}$ to have physical meaning, the condition $\beta>0$ must be satisfied. This was showed in [33]. Based on the GUP, the black-hole's remnant was first studied by Adler et al. [35]. Incorporating Eq. (3) into the Dirac equation in curved spacetime, the modified Dirac equation was derived [32]. Using this modified equation, the fermions' tunneling from the Schwarzschild spacetime was investigated. The temperature was showed to be related to the quantum numbers of the emitted fermion. An interesting result is that the quantum correction slows down the increase of the temperature. It naturally is to lead to a remnant.

In this paper, taking into account the effects of quantum gravity, we investigate fermions' tunneling from a fivedimensional rotating black string. The key point in this paper is to construct a tetrad and five gamma matrices. The result shows that in the frame of quantum gravity, the temperature is affected not only by the quantum numbers of the emitted fermion, but also by the effect of the extra compact dimension. The quantum correction slows down the increase of the temperature. A remnant is naturally observed in the evaporation process.

In Sect. 2, we perform the dragging coordinate transformation on the metric and construct five gamma matrices; then we investigate the fermion's tunneling from the five-dimensional rotating string. A remnant is observed. Section 3 is devoted to our conclusion.

\section{Tunneling radiation under the influence of the generalized uncertainty principle}

The Kerr metric describes a rotating black-hole solution of the Einstein equations in four dimensions. When we add an extra compact spatial dimension to it, the metric becomes

$$
\begin{aligned}
\mathrm{d} s^{2}= & -\frac{\Delta}{\rho^{2}}\left(\mathrm{~d} t-a \sin ^{2} \theta \mathrm{d} \varphi\right)^{2}+\frac{\sin ^{2} \theta}{\rho^{2}}\left[a \mathrm{~d} t-\left(r^{2}+a^{2}\right) \mathrm{d} \varphi\right]^{2} \\
& +\frac{\rho^{2}}{\Delta} \mathrm{d} r^{2}+\rho^{2} \mathrm{~d} \theta^{2}+g_{z z} \mathrm{~d} z^{2}
\end{aligned}
$$

where $\Delta=r^{2}-2 M r+a^{2}=\left(r-r_{+}\right)\left(r-r_{-}\right), \rho^{2}=r^{2}+$ $a^{2} \cos ^{2} \theta, g_{z z}$ is usually set to 1 . The above metric describes a rotating uniform black string. $r_{ \pm}=M \pm \sqrt{M^{2}-a^{2}}$ are the locations of the event (inner) horizons. $M$ and $a$ are the mass and angular momentum in units of mass of the string, respectively. A fermion's motion satisfies the generalized Dirac equation (1). To investigate the tunneling behavior of the fermion, it can directly choose a tetrad and construct gamma matrices from the metric (5). The metric (5) describes a rotating spacetime. The energy and mass near the horizons are dragged by the rotating spacetime. It is not convenient to discuss the fermion's tunneling behavior. For the convenience of constructing the tetrad and gamma matrices, we perform the dragging coordinate transformation $\mathrm{d} \phi=\mathrm{d} \varphi-\Omega \mathrm{d} t$, where

$\Omega=\frac{\left(r^{2}+a^{2}-\Delta\right) a}{\left(r^{2}+a^{2}\right)^{2}-\Delta a^{2} \sin ^{2} \theta}$,

on the metric (5). Then the metric (5) takes the form

$$
\begin{aligned}
\mathrm{d} s^{2}= & -F(r) \mathrm{d} t^{2}+\frac{1}{G(r)} \mathrm{d} r^{2}+g_{\theta \theta} \mathrm{d} \theta^{2}+g_{\phi \phi} \mathrm{d} \phi^{2}+g_{z z} \mathrm{~d} z^{2} \\
= & -\frac{\Delta \rho^{2}}{\left(r^{2}+a^{2}\right)^{2}-\Delta a^{2} \sin ^{2} \theta} \mathrm{d} t^{2}+\frac{\rho^{2}}{\Delta} \mathrm{d} r^{2}+g_{z z} \mathrm{~d} z^{2} \\
& +\rho^{2} \mathrm{~d} \theta^{2}+\frac{\sin ^{2} \theta}{\rho^{2}}\left[\left(r^{2}+a^{2}\right)^{2}-\Delta a^{2} \sin ^{2} \theta\right] \mathrm{d} \phi^{2} .
\end{aligned}
$$

Now the tetrad is directly constructed from the above metric. It is

$e_{\mu}^{a}=\operatorname{diag}\left(\sqrt{F}, 1 / \sqrt{G}, \sqrt{g_{\theta \theta}}, \sqrt{g_{\phi \phi}}, \sqrt{g_{z z}}\right)$.

The gamma matrices are easily constructed as follows:

$$
\begin{aligned}
& \gamma^{t}=\frac{1}{\sqrt{F}}\left(\begin{array}{ll}
0 & I \\
-I & 0
\end{array}\right), \quad \gamma^{\theta}=\sqrt{g^{\theta \theta}}\left(\begin{array}{ll}
0 & \sigma^{2} \\
\sigma^{2} & 0
\end{array}\right), \\
& \gamma^{r}=\sqrt{G}\left(\begin{array}{ll}
0 & \sigma^{3} \\
\sigma^{3} & 0
\end{array}\right), \quad \gamma^{\phi}=\sqrt{g^{\phi \phi}}\left(\begin{array}{ll}
0 & \sigma^{1} \\
\sigma^{1} & 0
\end{array}\right), \\
& \gamma^{z}=\sqrt{g^{z z}}\left(\begin{array}{ll}
-I & 0 \\
0 & I
\end{array}\right) .
\end{aligned}
$$

While measuring the quantum property of a spin-1/2 fermion, we can get two values. They correspond to two states with spin up and spin down. The wave functions of two states of a fermion in the metric (7) of the spacetime take on the form

$$
\begin{aligned}
& \psi_{(\uparrow)}=\left(\begin{array}{c}
A \\
0 \\
B \\
0
\end{array}\right) \exp \left(\frac{i}{\hbar} I_{\uparrow}(t, r, \theta, \phi, z)\right), \\
& \psi_{(\downarrow)}=\left(\begin{array}{l}
0 \\
C \\
0 \\
D
\end{array}\right) \exp \left(\frac{i}{\hbar} I_{\downarrow}(t, r, \theta, \phi, z)\right),
\end{aligned}
$$


where $A, B, C, D$ are functions of $(t, r, \theta, \phi, z)$, and $I$ is the action of the fermion, and $\uparrow$ and $\downarrow$ denote the spin up and spin down, respectively. In this paper, we only investigate the state with spin up. The analysis of the state with spin down is parallel. To use the WKB approximation, we insert the wave function (10) and the gamma matrices into the generalized Dirac equation (1). Dividing by the exponential term and considering the leading terms yield four equations. They are

$$
\begin{aligned}
& -\frac{B}{\sqrt{F}} \partial_{t} I-B \sqrt{G}\left(1-\beta m^{2}\right) \partial_{r} I+A \sqrt{g^{z z}}\left(1-\beta m^{2}\right) \partial_{z} I \\
& -A m\left(1-\beta m^{2}-\beta Q\right)+B \beta \sqrt{G} Q \partial_{r} I-A \beta \sqrt{g^{z z}} Q \partial_{z} I=0,
\end{aligned}
$$

$$
\begin{aligned}
& \frac{A}{\sqrt{F}} \partial_{t} I-A \sqrt{G}\left(1-\beta m^{2}\right) \partial_{r} I-B \sqrt{g^{z z}}\left(1-\beta m^{2}\right) \partial_{z} I \\
& -B m\left(1-\beta m^{2}-\beta Q\right)+A \beta \sqrt{G} Q \partial_{r} I+B \beta \sqrt{g^{z z}} Q \partial_{z} I=0 \\
& -B\left(i \sqrt{g^{\theta \theta}} \partial_{\theta} I+\sqrt{g^{\phi \phi}} \partial_{\phi} I\right)\left(1-\beta m^{2}-\beta Q\right)=0 \\
& -A\left(i \sqrt{g^{\theta \theta}} \partial_{\theta} I+\sqrt{g^{\phi \phi}} \partial_{\phi} I\right)\left(1-\beta m^{2}-\beta Q\right)=0
\end{aligned}
$$

where $Q=g^{r r}\left(\partial_{r} I\right)^{2}+g^{\theta \theta}\left(\partial_{\theta} I\right)^{2}+g^{\phi \phi}\left(\partial_{\phi} I\right)^{2}+$ $g^{z z}\left(\partial_{z} I\right)^{2}$. It is difficult to get the expression of the action from the above equations. Considering the property of the spacetime, we carry out separation of the variables as

$I=-(\omega-j \Omega) t+W(r)+\Theta(\theta, \phi)+J z$

where $\omega$ is the energy of the emitted fermion, $j$ is the angular momentum and $J$ is a conserved momentum corresponding to the compact dimension. Equations (14) and (15) are irrelevant to $A, B$. Inserting Eq. (16) into them yields

$i \sqrt{g^{\theta \theta}} \partial_{\theta} \Theta+\sqrt{g^{\phi \phi}} \partial_{\phi} \Theta=0$,

which implies that $\Theta$ is a complex function other than the constant solution. In the former research, it was found that the contribution of $\Theta$ could be canceled in the derivation of the tunneling rate. Using Eq. (17), the important relation is easily gotten of

$g^{\theta \theta}\left(\partial_{\theta} \Theta\right)^{2}+g^{\phi \phi}\left(\partial_{\phi} \Theta\right)^{2}=0$.

Now our interest is in the first two equations. Inserting Eq. (16) into Eqs. (12) and (13), canceling $A$ and $B$ and neglecting the higher order terms of $\beta$, we get

$A\left(\partial_{r} W\right)^{4}+B\left(\partial_{r} W\right)^{2}+C=0$,

where

$A=2 \beta G^{2} F$,

$B=-\left[1-4 \beta g^{z z}\left(\partial_{z} I\right)^{2}\right] G F$,

$C=\left[1-2 \beta m^{2}-2 \beta g^{z z}\left(\partial_{z} I\right)^{2}\right]\left(m^{2}-g^{z z}\left(\partial_{z} I\right)^{2}\right) F+\left(\partial_{t} I\right)^{2}$.
Solving the above equation at the event horizon yields the imaginary part of the radial action. Based on invariance under canonical transformations, we adopt the method developed in [39-41]. The tunneling rate is

$$
\begin{aligned}
\Gamma & \propto \exp \left[-\frac{1}{\hbar} \operatorname{Im} \oint p_{r} \mathrm{~d} r\right] \\
& =\exp \left[-\frac{1}{\hbar} \operatorname{Im}\left(\int p_{r}^{\text {out }} \mathrm{d} r-\int p_{r}^{\text {in }} \mathrm{d} r\right)\right] \\
& =\exp \left[\mp \frac{2}{\hbar} \operatorname{Im} \int p_{r}^{\text {out }, \text { in }} \mathrm{d} r\right] .
\end{aligned}
$$

In the above equation, $\oint p_{r} \mathrm{~d} r$ is invariant under canonical transformations. Here let us write $p_{r}=\partial_{r} W$. Thus the solutions of $\operatorname{Im} \int p_{r}^{\text {out,in }} \mathrm{d} r$ are determined by Eq. (19), which is

$$
\begin{aligned}
\operatorname{Im} & \oint p_{r} \mathrm{~d} r=2 \operatorname{Im} W^{\text {out }} \\
= & 2 \operatorname{Im} \int \mathrm{d} r \sqrt{\frac{(E-j \Omega)^{2}+\left(1-2 \beta m^{2}-2 \beta g^{z z} J^{2}\right)\left(m^{2}-g^{z z} J^{2}\right) F}{G F\left(1-4 \beta g z z J^{2}\right)}} \\
& \times\left[1+\beta\left(\frac{(E-j \Omega)^{2}}{F}+m^{2}-g^{z z} J^{2}\right)\right] \\
= & 2 \pi \frac{\left(\omega-j \Omega_{+}\right)\left(r_{+}^{2}+a^{2}\right)}{r_{+}-r_{-}}\left[1+\beta \Xi\left(J, \theta, r_{+}, j\right)\right],
\end{aligned}
$$

where $g^{z z}=1, \Omega_{+}=\frac{a}{r_{+}^{2}+a^{2}}$ is the angular velocity at the event horizon. $\Xi\left(J, \theta, r_{+}, j\right)$ is a complicated function of $J, \theta, r_{+}, j$; therefore, we do not write it down here. We should have $\Xi\left(J, \theta, r_{+}, j\right)>0$. If we adopt Eq. (22) to calculate the tunneling rate, we will derive a twice higher Hawking temperature, which was showed in [36-38]. This is not in consistence with the standard temperature. With careful observations, Akhmedova et al. [39-41] found that the contribution coming from the temporal part of the action was ignored. When they took into account the temporal contribution, the factor of 2 in the temperature was resolved.

To find the temporal contribution, we use Kruskal coordinates, $(T, R)$. The region exterior to the string $\left(r>r_{+}\right)$is described by

$$
T=e^{\kappa_{+} r_{*}} \sinh \left(\kappa_{+} t\right),
$$$$
R=e^{\kappa_{+} r_{*}} \cosh \left(\kappa_{+} t\right),
$$

where $r_{*}=r+\frac{1}{2 \kappa_{+}} \ln \frac{r-r_{+}}{r_{+}}-\frac{1}{2 \kappa_{-}} \ln \frac{r-r_{-}}{r_{-}}$is the tortoise coordinate, and $\kappa_{ \pm}=\frac{r_{+}-r_{-}}{2\left(r_{ \pm}^{2}+a^{2}\right)}$ denote the surface gravity at the outer (inner) horizons. The description of the interior region is given by

$$
\begin{aligned}
& T=e^{\kappa_{+} r_{*}} \cosh \left(\kappa_{+} t\right), \\
& R=e^{\kappa_{+} r_{*}} \sinh \left(\kappa_{+} t\right) .
\end{aligned}
$$

To connect these two patches across the horizon, we need to rotate the time $t$ as $t \rightarrow t-i \kappa_{+} \frac{\pi}{2}$. As pointed out in [39-41], this rotation would lead to an additional imagi- 
nary contribution coming from the temporal part, namely $\operatorname{Im}\left(E \Delta t^{\text {out,in }}\right)=\frac{1}{2} \pi E \kappa_{+}$, where $E=\omega-j \Omega_{+}$. Thus the total temporal contribution is $\operatorname{Im}(E \Delta t)=\pi E \kappa_{+}$. Therefore, the tunneling rate is

$$
\begin{aligned}
\Gamma & \propto \exp \left[-\frac{1}{\hbar}\left(\operatorname{Im}(E \Delta t)+\operatorname{Im} \oint p_{r} \mathrm{~d} r\right)\right] \\
& =-4 \pi \frac{\left(\omega-j \Omega_{+}\right)\left(r_{+}^{2}+a^{2}\right)}{\hbar\left(r_{+}-r_{-}\right)}\left[1+\frac{1}{2} \beta \Xi\left(J, \theta, r_{+}, j\right)\right] .
\end{aligned}
$$

This is the expression of the Boltzman factort and it implies for the temperature

$$
\begin{aligned}
T & =\frac{\hbar\left(r_{+}-r_{-}\right)}{4 \pi\left(r_{+}^{2}+a^{2}\right)\left[1+\frac{1}{2} \beta \Xi\left(J, \theta, r_{+}, j\right)\right]} \\
& =T_{0}\left[1-\frac{1}{2} \beta \Xi\left(J, \theta, r_{+}, j\right)\right],
\end{aligned}
$$

where $T_{0}=\frac{\hbar\left(r_{+}-r_{-}\right)}{4 \pi\left(r_{+}^{2}+a^{2}\right)}$ is the standard Hawking temperature of the Kerr string; it shares the expression of the temperature with the four-dimensional Kerr black hole. It shows that the corrected temperature is determined by the mass, angular momentum and extra dimension of the string, but also it is affected by the quantum numbers (energy, mass, and angular momentum) of the fermion. Therefore, the properties of the emitted fermion affect the temperature when the effects of quantum gravity are taken into account.

When $a=0$, the metric (5) is reduced to the Schwarzschild string metric. Then the imaginary part of the radial action (22) is reduced to

$\operatorname{Im} \oint p_{r} \mathrm{~d} r=2 \pi \omega r_{+}\left[1+\beta\left(2 \omega^{2}+3 m^{2} / 2+J^{2} / 2\right)\right]$.

Adopting the same process, we get the temperature of the Schwarzschild string:

$$
\begin{aligned}
T & =\frac{\hbar}{4 \pi r_{+}\left[1+\beta\left(\omega^{2}+3 m^{2} / 4+J^{2} / 4\right)\right]} \\
& =\frac{\hbar}{8 \pi M}\left[1-\beta\left(\omega^{2}+3 m^{2} / 4+J^{2} / 4\right)\right] .
\end{aligned}
$$

It shows that the effect of the extra dimension and the quantum numbers (energy, mass, and angular momentum) of the fermion affect the temperature of the Schwarzschild string. It is obvious that the quantum correction slows down the increase of the temperature. Finally, the string cannot evaporate completely and in the end there is a balanced state. In this state, a remnant is left. The extra dimension plays the role of an impediment during the evaporation. When $J=0$, Eq. (28) describes the temperature of the four-dimensional Schwarzschild black hole. The remnant was derived as $\geq$ $M_{p} / \beta_{0}$, where $M_{p}$ is the Planck mass and $\beta_{0}$ is a dimensionless parameter accounting for quantum gravity effects [32].

\section{Conclusion}

In this paper, we investigated the fermion's tunneling from the five-dimensional Kerr string spacetime. To incorporate the influence of quantum gravity, we adopted the generalized Dirac equation derived in [32]. The corrected temperature is not only determined by the mass, angular momentum, and extra dimension, but also it is affected by the quantum numbers of the emitted fermion. The quantum correction slows down the increase of the temperature. Finally, a balanced state appears. In this state, the string cannot evaporate completely and a remnant is left. This can be seen as a direct consequence of the generalized uncertainty principle.

Acknowledgments This work is supported by the National Natural Science Foundation of China with Grant No. 11205125.

Open Access This article is distributed under the terms of the Creative Commons Attribution License which permits any use, distribution, and reproduction in any medium, provided the original author(s) and the source are credited.

Article funded by $\mathrm{SCOAP}^{3}$ and licensed under CC BY 4.0

\section{References}

1. P. Kraus, F. Wilczek, Nucl. Phys. B 437, 231 (1995)

2. P. Kraus, F. Wilczek, Nucl. Phys. B 433, 403 (1995)

3. M.K. Parikh, F. Wilczek, Phys. Rev. Lett. 85, 5042 (2000)

4. M.K. Parikh, Phys. Lett. B 546, 189 (2002)

5. S.W. Hawking, Commun. Math. Phys. 43, 199 (1975)

6. T. Damour, R. Ruffini, Phys. Rev. D 14, 332 (1976)

7. W.G. Unruh, Phys. Rev. D 14, 870 (1976)

8. S.P. Robinson, F. Wilczek, Phys. Rev. Lett. 95, 011303 (2005)

9. S. Iso, H. Umetsu, F. Wilczek, Phys. Rev. Lett. 96, 151302 (2006)

10. P. Mitra, Phys. Lett. B 648, 240 (2007)

11. E.C. Vagenas, Phys. Lett. B 533, 302 (2002)

12. M. Arzano, A.J.M. Medved, E.C. Vagenas, JHEP 0509, 037 (2005)

13. S.Q. Wu, Q.Q. Jiang, JHEP 0603, 079 (2006)

14. S.Z. Yang, Chin. Phys. Lett. 22, 2492 (2005)

15. Y.P. Hu, J.Y. Zhang, Z. Zhao, Int. J. Mod. Phys. D 16, 847 (2007)

16. J.Y. Zhang, Z. Zhao, JHEP 0510, 055 (2005)

17. J.Y. Zhang, Z. Zhao, Nucl. Phys. B 725, 173 (2005)

18. Q.Q. Jiang, S.Q. Wu, X. Cai, Phys. Rev. D 73, 064003 (2006)

19. R. Kerner, R.B. Mann, Class. Quant. Grav. 25, 095014 (2008)

20. R. Kerner, R.B. Mann, Phys. Lett. B 665, 277 (2008)

21. M. Angheben, M. Nadalini, L. Vanzo, S. Zerbini, JHEP 0505, 014 (2005)

22. K. Srinivasan, T. Padmanabhan, Phys. Rev. D 60, 024007 (1999)

23. R. Li, J.R. Ren, Phys. Lett. B 661, 370 (2008)

24. R.D. Criscienzo, L. Vanzo, Europhys. Lett. 82, 60001 (2008)

25. Q.Q. Jiang, Phys. Rev. D 78, 044009 (2008)

26. K. Lin, S.Z. Yang, Phys. Rev. D 79, 064035 (2009)

27. R. Banerjee, B.R. Majhi, JHEP 0806, 095 (2008)

28. B.R. Majhi, Phys. Rev. D 79, 044005 (2009)

29. D. Singleton, E.C. Vagenas, T. Zhu, J.R. Ren, JHEP 1008, 089 (2010)

30. K. Nozari, S. Saghafi, JHEP 1211, 005 (2012)

31. K. Nozari, S.H. Mehdipour, JHEP 0903, 061 (2009)

32. D. Chen, H. Wu and H. Yang, Fermions tunnelling with effects of quantum gravity. arXiv:1305.7104 [gr-qc] 
33. A. Kempf, G. Mangano, R.B. Mann, Phys. Rev. D 52, 1108 (1995)

34. S. Das, E.C. Vagenas, Phys. Rev. Lett. 101, 221301 (2008)

35. R.J. Adler, P. Chen, D.I. Santiago, Gen. Relativ. Gravit. 33, 2101 (2001)

36. E.T. Akhmedov, V. Akhmedova, T. Pilling, D. Singleton, Int. J. Mod. Phys. A 22, 1705 (2007)

37. B.D. Chowdhury, Pramana 70, 593 (2008)

38. E.T. Akhmedov, V. Akhmedova, D. Singleton, Phys. Lett. B 642, 124 (2006)
39. V. Akhmedova, T. Pilling, A. de Gill, D. Singleton, Phys. Lett. B 666, 269 (2008)

40. E.T. Akhmedov, T. Pilling, D. Singleton, Int. J. Mod. Phys. D 17, 2453 (2008)

41. V. Akhmedova, T. Pilling, A. de Gill, D. Singleton, Phys. Lett. B 673, 227 (2009) 